\title{
A 5V CLOSED-LOOP SECOND-ORDER SIGMA-DELTA MICRO-G MICROACCELEROMETER
}

\author{
Haluk Kulah, Arvind Salian", Navid Yazdi", and Khalil Najafi \\ Center for Wireless Integrated Microsystems \\ University of Michigan, Ann Arbor, MI 48109-2122 \\ email: haluk.kulah@umich.edu
}

\begin{abstract}
A closed-loop hybrid accelerometer subsystem consisting of an all-silicon $\mu \mathrm{g}$ capacitive microaccelerometer and a CMOS interface circuit is presented. Microaccelerometer sensitivities are $1.3 \mathrm{pF} / \mathrm{g}$ and $6 \mathrm{pF} / \mathrm{g}$ for $2 \times 1 \mathrm{~mm}^{2}$ and $4 \times 1 \mathrm{~mm}^{2}$ doubly-supported bridge structures, and $20 \mathrm{pF} / \mathrm{g}$ for a cantilever-supported structure, respectively. The calculated mechanical noise floor for the $2 \mathrm{x} 1 \mathrm{~mm}^{2}$ bridge device is $0.39 \mu \mathrm{g} / \mathrm{V} \mathrm{Hz}$ in atmosphere. The circuit has a $95 \mathrm{~dB}$ dynamic range, a low offset of $370 \mu \mathrm{V}$ and can resolve better than $75 \mathrm{aF}$. The complete module operates from a single $5 \mathrm{~V}$ supply and has a measured sensitivity of $430 \mathrm{mV} / \mathrm{g}$ with a noise floor of $15 \mu \mathrm{g} / \sqrt{\mathrm{Hz}}$ in open-loop. The subsystem is operated in closed-loop and can resolve better than $100 \mu \mathrm{g} / \sqrt{\mathrm{Hz}}$ for signal frequencies up to $15 \mathrm{~Hz}$, and better than $2 \mu \mathrm{g} / \sqrt{\mathrm{Hz}}$ for signal frequencies higher than $15 \mathrm{~Hz}$.
\end{abstract}

\section{INTRODUCTION}

Accelerometer systems with micro-g resolution and high precision are needed in navigation/guidance, microgravity measurements, tilt control, platform stabilization, and position sensing [1]. The sensor should have high sensitivity, low temperature sensitivity, and good long-term stability. In order to obtain a large dynamic range and reduce sensitivity to variations in accelerometer mechanical characteristics (mass size, spring constant), closed-loop operation based on force feedback is required. In addition, a digital output is desired in many applications. Our group has previously reported a highperformance silicon microaccelerometer [2], and its open-loop operation using a switched-capacitor readout circuit [3]. In this paper, we report the closed-loop operation of the accelerometer system as a second-order sigma-delta modulator using only a single $5 \mathrm{~V}$ supply.

\section{THE ACCELEROMETER SYSTEM}

To increase accelerometer sensitivity, a large proof mass, compliant suspensions, and large capacitance sensitivity are needed. When operated in open-loop, such an accelerometer will have a limited dynamic range and bandwidth. To increase the dynamic range, the accelerometer can be operated in closed-loop by applying an electrostatically-generated force feedback signal that maintains the proof mass in null position. Recently, $\Sigma-\Delta$ modulation control loops have been used in microaccelerometers to achieve force-rebalancing and obtain a direct digital output [3, 4]. A $2^{\text {nd }}$-order $\Sigma-\Delta$ modulator, with two integrators, one mechanical and one electrical, not only provides a digital output, but also relaxes the accuracy requirements on the analog circuits.

The general form of a $\Sigma-\Delta$ modulator is shown in Figure 1. The $\Sigma-\Delta$ loop has a low pass transfer function represented by $\mathrm{H}(\mathrm{s})$.
This low pass characteristic moves most of the quantization noise to higher frequencies out of the input signal band. The high frequency shaped noise is removed from the converted signal by the low pass digital filter following the $\Sigma-\Delta$ modulator loop. In an electromechanical $\Sigma-\Delta$ modulator, $H(\mathrm{~s})$ is realized by the low pass transfer function of the sensor. The oversampling ratio in electromechanical modulators is typically very large. Hence sufficiently low quantization noise is achievable with a one-bit digital-to-analog converter (DAC) and quantizer; therefore, the DAC block and the nonlinearity errors of the quantizer are reduced.

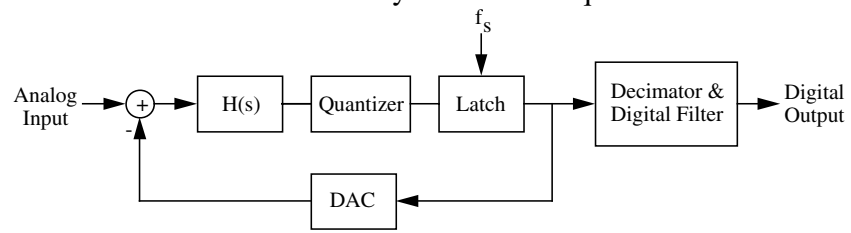

Figure 1: General oversampling sigma-delta data converter.

The electrostatic force-feedback for the accelerometer is provided by the high frequency pulse-width modulated (PWM) digital output stream of the electromechanical $\Sigma-\Delta$ modulator. Therefore the closed-loop accelerometer has all the advantages of an oversampled $\Sigma-\Delta$ data converter as well as direct digital output, very wide dynamic range, extended linearity, less stringent precision requirements of the circuit blocks, and larger bandwidth. All these advantages are achieved in an oversampled $\Sigma-\Delta$ modulator by trading-off resolution in the time domain with resolution of the various circuits. The relatively small bandwidth $(1 \mathrm{kHz})$, high precision ( $\mu \mathrm{g}$ 's) and wide dynamic range $(18-19 \mathrm{~b})$ requirements of our application, make this trade-off very rewarding.

The accelerometer system consists of the sensor and the interface chip. The sensor structure utilizes the whole wafer thickness to attain a large proof mass with integrated polysilicon electrodes above/below it [2]. These electrodes are stiffened by embedding vertical stiffeners in them to allow force rebalancing of the proof mass. Damping holes are formed in the electrodes to obtain a desired damping coefficient for stable operation. Figure 2 shows the device SEM with a $2 \times 1 \mathrm{~mm}^{2}$ proof mass.

In force-rebalanced accelerometers with a large proof mass, a further challenge is maintaining low-voltage operation since the required feedback force has to be large enough to bring the proof mass to null position. In the system reported here, this is accomplished by using a single 5V supply, and by limiting the movement of the proof mass using bushings formed between the electrodes and proof mass as illustrated in Figure 3.

The electrostatic feedback force is a linear function of the output voltage and is linearized by applying differential force on top and bottom electrodes. Assuming that the proof mass

\footnotetext{
* Now with: Motorola Inc., Phoenix, AZ

${ }^{* * *}$ Now with: Corning Intellisense Corp., Wilmington, MA 01887
} 
displacement is negligible in closed-loop operation, if the circuit applies $\mathrm{V}_{0}+\mathrm{V} / 2$ on the top and $\mathrm{V}_{0}-\mathrm{V} / 2$ on the bottom electrodes, the differential electrostatic force can be expressed as:

$$
F_{e-\text { diff }}=F_{e-\text { top }}-F_{e-\text { bottom }} \approx \frac{\varepsilon_{0} A}{d^{2}} V_{0} V
$$

where A is the electrode area and $\mathrm{d}$ is the gap distance between the electrode and the proof mass. It can be shown that the full-scale feedback acceleration is:

$$
a_{f s}=\frac{1}{9.8} \times \frac{1}{m} \times \frac{T_{f}}{T} \times\left(\frac{\varepsilon_{0} A}{d^{2}} V_{0} V\right)
$$

where $T_{f} / T$ is the ratio of feedback duration over the full readout cycle, and $\mathrm{m}$ is the proof mass. For $\mathrm{m}=2.2 \mathrm{mg}, \mathrm{T}_{\mathrm{r}} \mathrm{T}=3 / 8, \mathrm{~A}=1.4 \mathrm{~mm}^{2}$ (effective electrode area after taking the damping holes into account), $\mathrm{d}=1.5 \mu \mathrm{m}$ and $\mathrm{V}=2 \mathrm{~V}_{0}=5 \mathrm{volts}$, the maximum feedback acceleration is $1.2 \mathrm{~g}$. In other words, this system can forcerebalance such a large proof mass by a single $5 \mathrm{~V}$ supply.

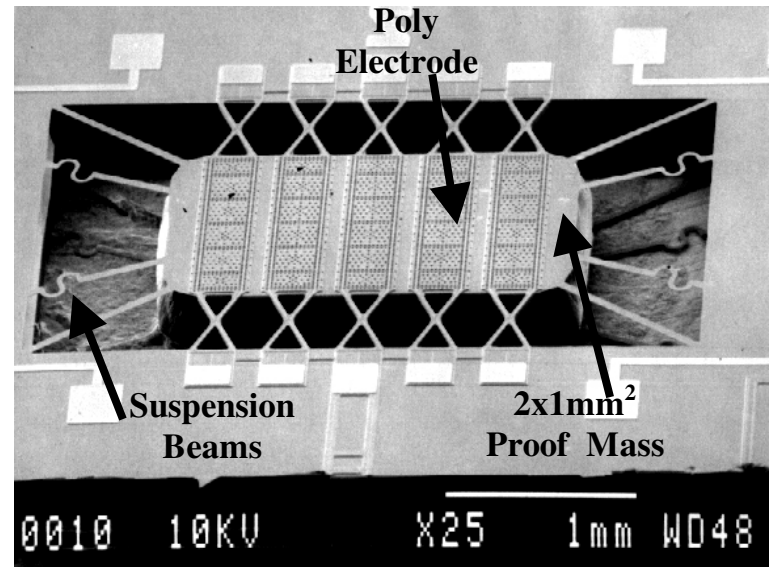

Figure 2. SEM of a device with $2 \mathrm{~mm} \times 1 \mathrm{~mm}$ proof mass.

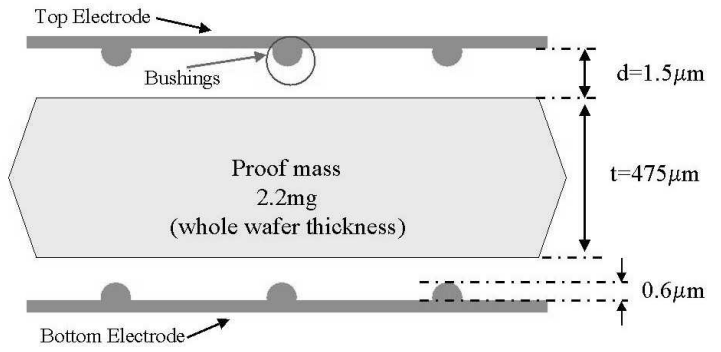

Figure 3: Cross sectional view of the accelerometer showing the electrodes, proof mass and bushings.

The microaccelerometer is interfaced with capacitive readout circuitry to detect the capacitance change and to operate the sensor in open-loop or force-rebalance the proof mass in closed-loop. Figure 4 shows the block diagram of the interface circuit [5]. Two fixed reference capacitors are used to form a balanced full-bridge with the sensor capacitive half-bridge, and the sensor top and bottom electrodes are used as the input nodes to the chip front-end. A switched-capacitor readout circuit is utilized to sense the capacitance change. This is because of the large parasitic capacitance associated with the hybrid packaging approach, and because of the large zero-acceleration sense capacitance associated with the accelerometer.

In addition to the switched-capacitor readout circuit, the interface chip incorporates two other important circuits. First, the start-up circuit pulls the proof mass to null position halfway between the two electrodes. At start-up, the proof mass is touching the bushings on the bottom electrodes, and hence the gap distance is approximately $2.4 \mu \mathrm{m}$. Because of the larger gap and in order to ensure that sufficient electrostatic force is available to bring the mass into null position, a start-up circuit is designed and used. This circuit stops the readout clock and applies a constant feedback voltage of 5 volts on the suitable electrode. The $5 \mathrm{~V}$ electrostatic signal can overcome the gravitational force of about $1.5 \mathrm{~g}$ from the proof mass. The design of the start-up circuit has to be such that the proof mass does not oscillate.

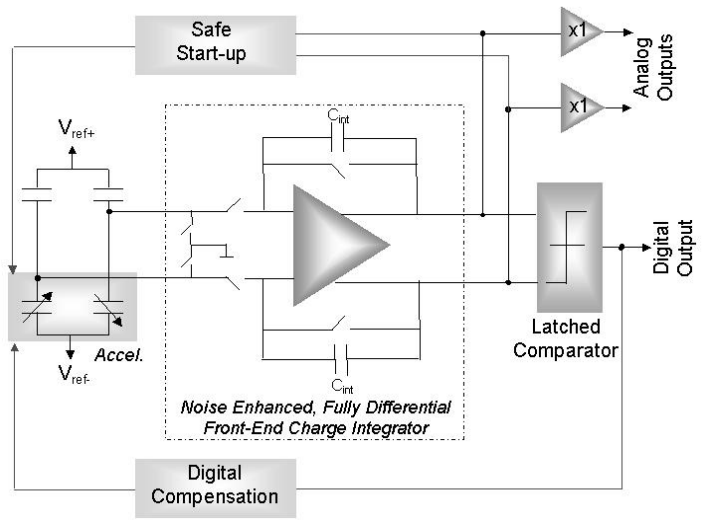

Figure 4: Block diagram of the interface sigma-delta chip.

Second, a digital feedback loop and digital compensation block are incorporated to provide a PWM signal to the proof mass and electrodes for force feedback operation. The digital compensation block implements a lead compensator to ensure the loop stability of the system, which degrades as the Q of the accelerometer increases. Electromechanical simulations have been performed to verify the stability of the system with an accelerometer Q of up to eight. Since the Q of the accelerometer implemented in our system is less than 1, the lead compensation circuit is usually not needed in normal operation. This lead compensator is realized by pulse-width modulating the feedback bit, which works quite effectively with the continuous time mechanical low pass transfer function. This approach provides better linearity without any need to implement the digital compensator in the forward signal path.

\section{TEST SETUP \& RESULTS}

Figure 5 shows the $\mathrm{z}$-axis hybrid subsystem with the sensor and the circuit assembled onto a PC board and mounted inside a standard DIP package. The sensor and the interface circuit are packaged close to each other to minimize parasitics. The hybrid system can operate in both open-loop and closed-loop.

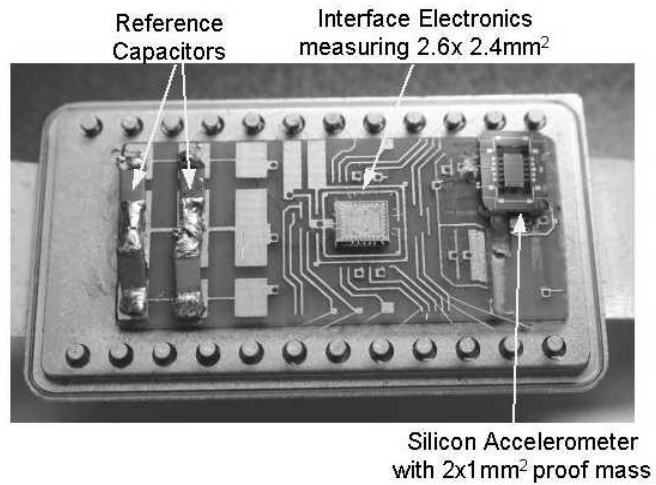

Figure 5: Hybrid packaged accelerometer and the interface chip in a standard 24-pin IC package. 


\section{Open-Loop Tests:}

The open-loop tests were done on a dividing head, in a $1 \mathrm{~g}$ gravitational field, by changing the acceleration on the sensor from $-1 \mathrm{~g}$ to $+1 \mathrm{~g}$. While changing the applied acceleration, both top and bottom capacitances were measured, as well as the analog output voltage of the interface electronics. Figure 6 shows the measured capacitance sensitivity for a $2 \times 1 \mathrm{~mm}^{2}$ bridge-type $\mathrm{z}$-axis accelerometer. As the figure shows, the variations of the top and bottom capacitances are perfectly symmetric with an offset of $8 \mathrm{pF}$ which is due to the difference in top and bottom gap distances. The offset is fabrication related and can be compensated in the test setup by using proper capacitance values while forming the full bridge. Measured sensitivities are $1.3 \mathrm{pF} / \mathrm{g}$ and $6 \mathrm{pF} / \mathrm{g}$ for $2 \times 1 \mathrm{~mm}^{2}$ and $4 \times 1 \mathrm{~mm}^{2}$ doubly-supported bridge structures, and $20 \mathrm{pF} / \mathrm{g}$ for a cantilever-supported structure, respectively.

The packaged accelerometer system is mounted on a PC board that includes sample-and-hold and low-pass-filter circuits to reduce the test setup related noise.

Figure 7 shows a measured open-loop sensitivity of $430 \mathrm{mV} / \mathrm{g}$. The noise floor is measured using a dynamic signal analyzer HP3561. Figure 8 shows the measured output noise spectrum of the complete module with a DC input of $1 \mathrm{~g}$, which shows a noise floor of $-100.3 \mathrm{~dB}$. This indicates that the current system is capable of resolving about $15 \mu \mathrm{g} / \sqrt{ } \mathrm{Hz}$.

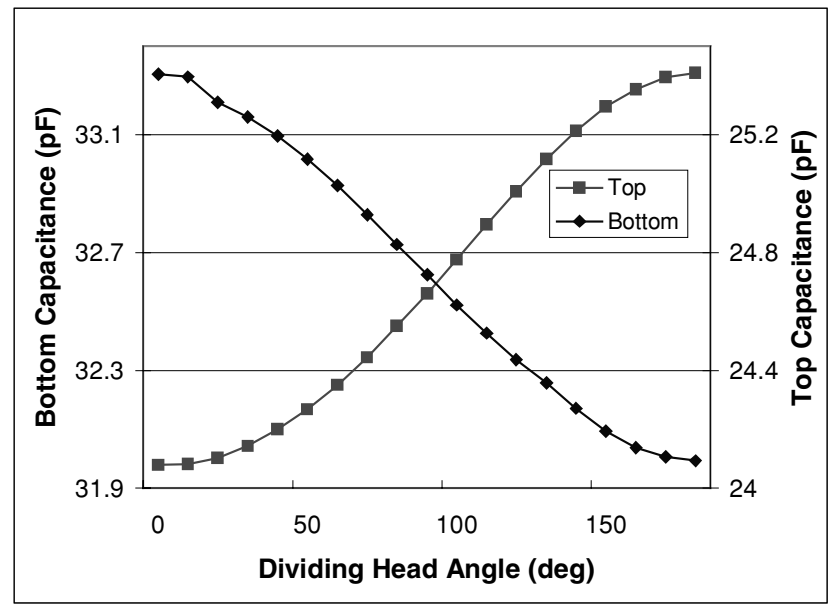

(a)

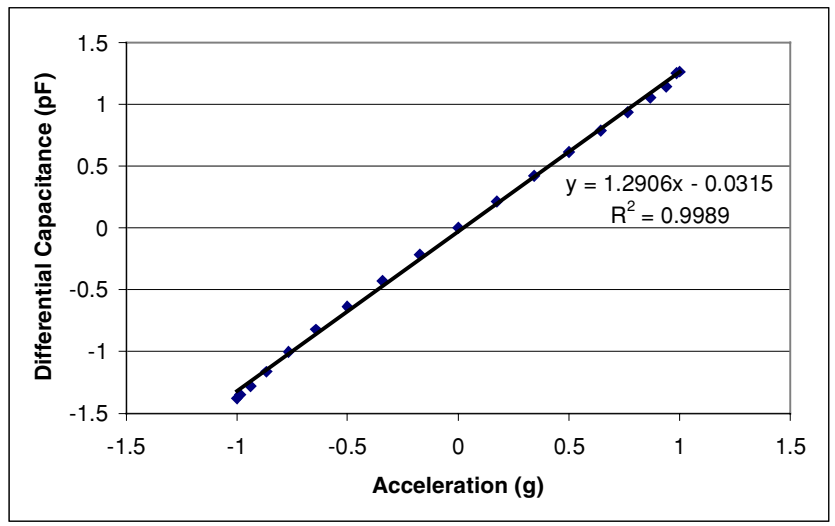

(b)

Figure 6: Measured capacitance sensitivity for the device with $2 \times 1 \mathrm{~mm}^{2}$ proofmass indicating a sensitivity of $1.3 \mathrm{pF} / \mathrm{g}$. (a) Top and Bottom electrodes capacitance variation for 180 degree rotation in earth's gravitational field. (b) Differential capacitance variation between -1 and $+1 g$.

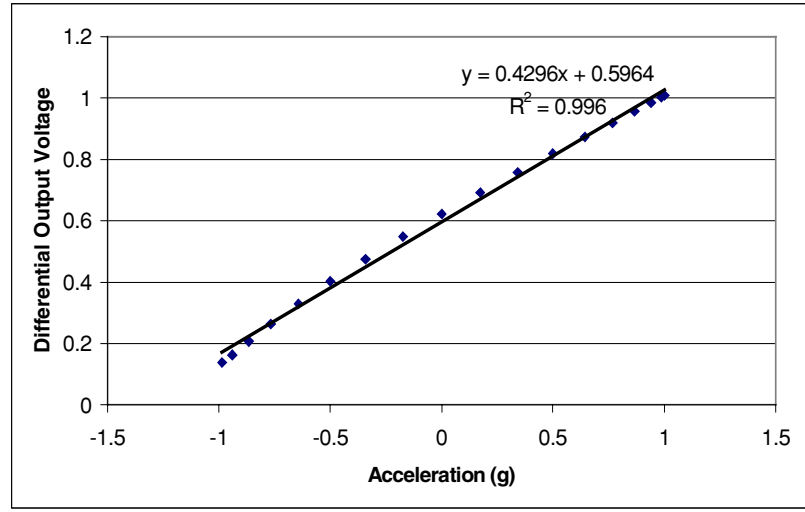

Figure 7: Measured open loop results for the subsystem with $2 \times 1 \mathrm{~mm}^{2}$ full-bridge type device. Overall sensitivity is $430 \mathrm{mV} / \mathrm{g}$.

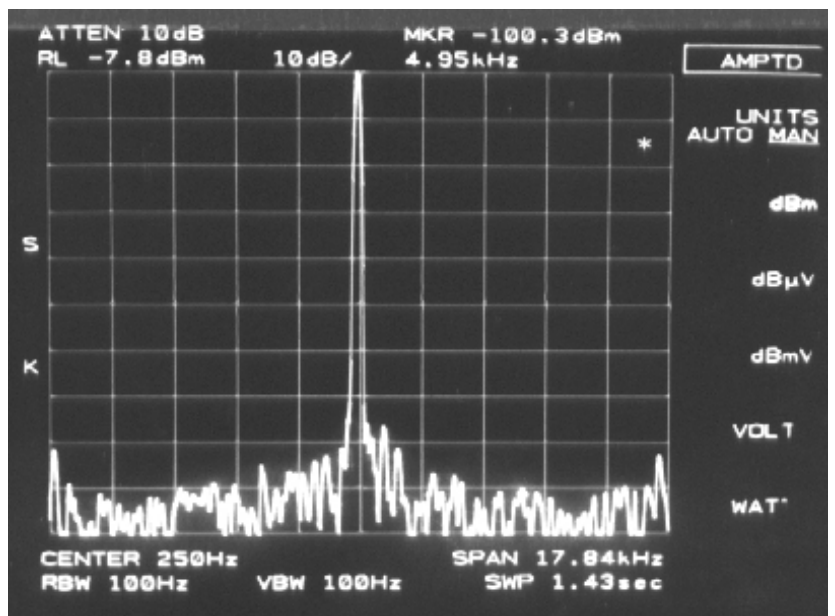

Figure 8: Noise measurement for the sub system indicating a resolution of $15 \mu \mathrm{g} / \sqrt{ } \mathrm{Hz}$.

\section{Closed-Loop Tests:}

Since the interface electronics uses a high oversampling sigma-delta modulation technique, the PWM output bit stream has to be processed to obtain an interpretable signal. This is realized by transferring the digital output to a computer by means of a data acquisition board, and processing the signal (decimating and digital filtering). A $\operatorname{sinc}^{3}$ filter, FIR filter, decimator and D/A converter have been implemented in MATLAB for this purpose. The closedloop test setup uses a shaker table, a data acquisition board and LABVIEW and MATLAB programs specifically written for this purpose.

The entire system has been operated closed-loop and the functionality of the system has been verified through extensive tests. Figure 9 shows the decimated PWM digital outputs for (a) pure $1 \mathrm{~g}$ DC input and (b) $0.25 \mathrm{~g}$ sinusoidal input acceleration on top of a $1 \mathrm{~g}$ DC. As the figure shows, the applied input acceleration is recovered successfully. Note that in Fig. 9(a), the only applied acceleration is the $1 \mathrm{~g}$ gravitational field. The output voltage is constant, except for variations due to noise generated in the system and/or picked up from the environment.

The recorded digital data was signal processed and the Fourier transform of the extracted signal was taken. Figure 10 shows the Fourier transform of the extracted signal for $1 \mathrm{~g}$ DC bias up to a frequency of $20 \mathrm{~Hz}$. As the figure shows, three regions can be identified. Up to a frequency of about $6 \mathrm{~Hz}$ the noise is slightly less than $100 \mu \mathrm{g} / \sqrt{\mathrm{Hz}}$. From $6 \mathrm{~Hz}$ to $16 \mathrm{~Hz}$, the noise reduces to about $2 \mu \mathrm{g} / \sqrt{ } \mathrm{Hz}$ and is constant after that. So, the overall resolution 
depends on the bandwidth and the range of frequencies of interest. The reasons for the higher noise at lower frequencies are not known yet.

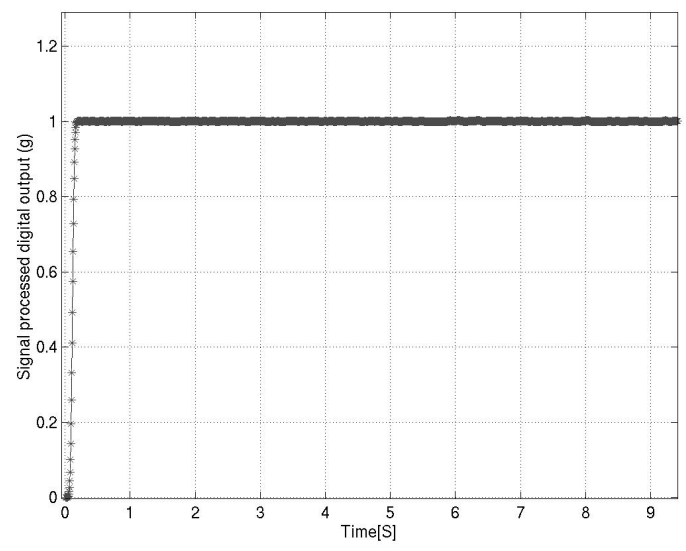

(a)

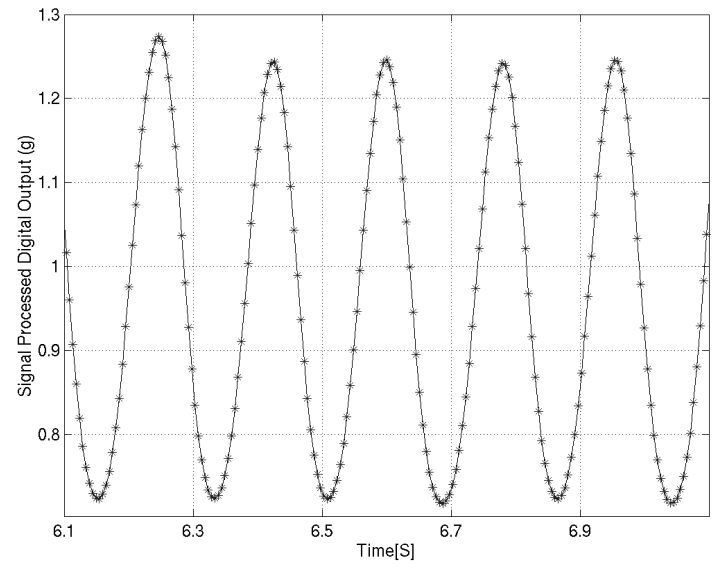

(b)

Figure 9: Closed-loop measurement results for the hybrid sensor subsystem: (a) for $1 \mathrm{~g}$ DC input acceleration, (b) for $0.25 \mathrm{~g}$ sinusoidal input acceleration on top of $1 \mathrm{~g} D C$ input.

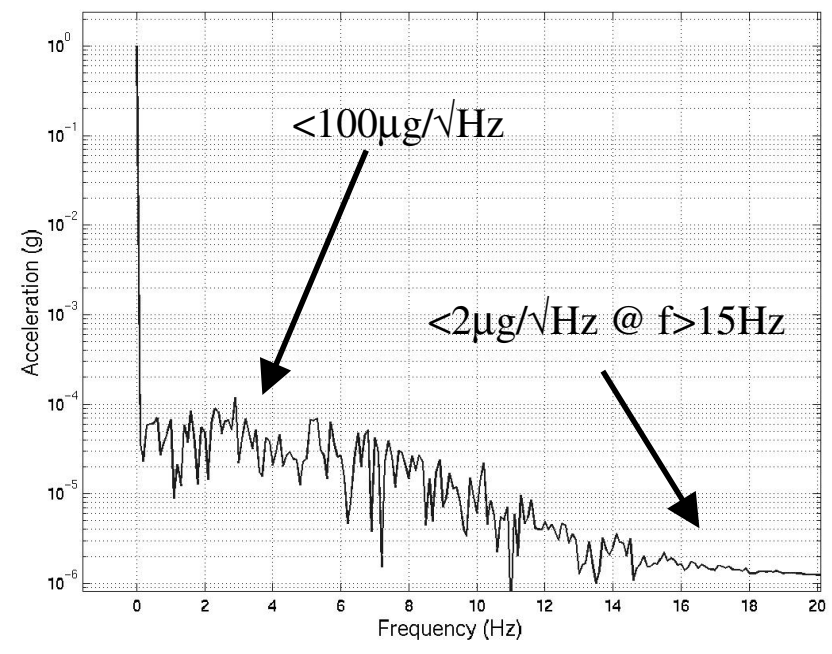

Figure 10: Closed-loop noise measurement results for $2 \mathrm{~mm} x$ $1 \mathrm{~mm}$ bridge structure under $1 \mathrm{~g}$ DC bias. Notice that the noise floor is less than $100 \mu \mathrm{g} / \sqrt{ } \mathrm{Hz}$.

\section{CONCLUSIONS}

A high performance $\mu \mathrm{g}$ accelerometer with high sensitivity, low noise, and closed-loop operation is presented. The accelerometer and the interface electronics operates as a $2^{\text {nd }}$ order electromechanical $\Sigma-\Delta$ modulator. Measured sensitivities are $1.3 \mathrm{pF} / \mathrm{g}$ and $6 \mathrm{pF} / \mathrm{g}$ for $2 \times 1 \mathrm{~mm}^{2}$ and $4 \times 1 \mathrm{~mm}^{2}$ doubly supported bridge structures, and $20 \mathrm{pF} / \mathrm{g}$ for a cantilever-supported structure respectively. The calculated mechanical noise floor for the device is $0.39 \mu \mathrm{g} / \sqrt{\mathrm{Hz}}$ at atmosphere. The circuit has a $95 \mathrm{~dB}$ dynamic range, a low offset of $370 \mu \mathrm{V}$ and can resolve better than $75 \mathrm{aF}$. The complete module has a measured sensitivity of $430 \mathrm{mV} / \mathrm{g}$ and a noise floor of $15 \mu \mathrm{g} / \mathrm{V} \mathrm{Hz}$ in open-loop. The closed loop operation of the system has been achieved for the first time and it has been shown that it results in a resolution better than $100 \mu \mathrm{g} / \mathrm{VHz}$ with a dynamic range of $82 \mathrm{~dB}$.

Table 1. Summary of the circuit/sensor performance

\begin{tabular}{|c|c|}
\hline \multicolumn{2}{|c|}{ Sensor Parameters } \\
\hline Sensitivity & $\begin{array}{ll}1.3 \mathrm{pF} / \mathrm{g} & \left(2 \mathrm{x} 1 \mathrm{~mm}^{2} \text { bridge }\right) \\
6 \mathrm{pF} / \mathrm{g} & \left(4 \mathrm{x} 1 \mathrm{~mm}^{2} \text { bridge }\right) \\
20 \mathrm{pF} / \mathrm{g} & \left(2 \mathrm{x} 1 \mathrm{~mm}^{2} \text { cant. }\right)\end{array}$ \\
\hline Mechanical Noise & $0.39 \mu \mathrm{g} / \sqrt{ } \mathrm{Hz}$ \\
\hline \multicolumn{2}{|c|}{ Interface IC Parameters } \\
\hline Sampling clock & $200 \mathrm{kHz}$ \\
\hline Power dissipation & $<6.6 \mathrm{~mW} @ 5 \mathrm{~V}$ \\
\hline Capacitance sensitivity & $0.3-1.1 \mathrm{~V} / \mathrm{pF}$ (adjustable) \\
\hline Noise floor & $85 \mu \mathrm{V} / \sqrt{\mathrm{Hz}} @ 1.1 \mathrm{~V} / \mathrm{pF}$ \\
\hline Offset & $2.7 \mathrm{mV}(370 \mu \mathrm{V}$ w/ chopper stab. $)$ \\
\hline \multicolumn{2}{|c|}{ Sensor and Interface Module } \\
\hline Sensitivity & $430 \mathrm{mV} / \mathrm{g}$ \\
\hline Open-loop resolution & $\sim 15 \mu \mathrm{g} / \sqrt{\mathrm{Hz}}$ (measured) \\
\hline Full scale range & $\pm 1.2 \mathrm{~g}$ with $5 \mathrm{~V}$ supply \\
\hline Closed-loop MDS & $100 \mu \mathrm{g} / \sqrt{ } \mathrm{Hz}$ \\
\hline
\end{tabular}

\section{ACKNOWLEDGMENTS}

The authors acknowledge the help of Mr. Junseok Chae, Mr. Brendan Casey, Mr. Robert Gordenker, and the staff of WIMS. This work has been supported by DARPA under contract F3060298-2-0231. Travel support has been provided by the Transducers Research Foundation and by the DARPA MEMS and DARPA BioFlips programs.

\section{REFERENCES}

1. N. Yazdi, F. Ayazi, and K. Najafi, "Micromachined Inertial Sensors," Proc. IEEE, vol. 86, no. 8, pp. 1640-1659, Aug.1998.

2. N. Yazdi, K. Najafi, "An All-Silicon Single-Wafer Micro-g Accelerometer with a Combined Surface and Bulk Micromachining Process," IEEE/ASME JMEMS, vol. 9, no. 4, pp. 1-8, Dec. 2000.

3. A. Salian, H. Kulah, N.Yazdi, G. He, and K. Najafi, "A HighPerformance Hybrid CMOS Microaccelerometer," In Tech. Dig. Solid-State Sensors and Actuators Workshop, Hilton Head Island, SC, USA, pp. 285-288, June 2000.

4. C. Lu, M. Lemkin, and B. Boser, "A monolithic surface micromachined accelerometer with digital output," IEEE J. Solid-State Circuits, vol. 30, no. 12, pp. 1367-1373, Dec. 1995.

5. N. Yazdi and K. Najafi, "An Interface IC for A Capacitive Silicon $\mu \mathrm{g}$ Accelerometer," Tech. Digest, 1999 IEEE ISSCC'99, Feb. 1999. 